Revue

Revue de l'histoire des religions

de Ihistoire

des religions

$1 \mid 2006$

Varia

Corpus de prières grecques et romaines, textes réunis, traduits et commentés par Frédéric Chapot et Bernard Laurot

Turnhout, Brepols, 2001

Marie-Karine Lhommé

\title{
CpenEdition
}

Journals

Édition électronique

URL : http://journals.openedition.org/rhr/4613

DOI : $10.4000 /$ rhr.4613

ISSN : 2105-2573

Éditeur

Armand Colin

Édition imprimée

Date de publication : 1 mars 2006

Pagination : 97-99

ISBN : 2200-92103-9

ISSN : 0035-1423

Référence électronique

Marie-Karine Lhommé, «Corpus de prières grecques et romaines, textes réunis, traduits et commentés par Frédéric Chapot et Bernard Laurot », Revue de l'histoire des religions [En ligne], 1 | 2006, mis en ligne le 18 janvier 2010, consulté le 22 septembre 2020. URL : http://journals.openedition.org/rhr/4613 ; DOI : https://doi.org/10.4000/rhr.4613

Ce document a été généré automatiquement le 22 septembre 2020

Tous droits réservés 


\section{Corpus de prières grecques et romaines, textes réunis, traduits et commentés par Frédéric Chapot et Bernard Laurot}

Turnhout, Brepols, 2001

Marie-Karine Lhommé

\section{RÉFÉRENCE}

Corpus de prières grecques et romaines, textes réunis, traduits et commentés par Frédéric Chapot et Bernard Laurot, Turnhout, Brepols, 2001, 24 cm, 446 p. («Recherches sur les rhétoriques religieuses ", 2), $67 €$.

1 Cet ouvrage est le deuxième de la collection «Recherches sur les Rhétoriques Religieuses ", et fait suite à la Bibliographie analytique de la prière grecque et romaine qui proposait des résumés d'articles et d'ouvrages, datant de 1898 à 1998, rangés par ordre alphabétique du nom de l'auteur. Cette fois ce sont les sources primaires païennes qui sont à l'honneur, avec un choix de 200 textes grecs et latins, au caractère littéraire plus ou moins marqué, bénéficiant d'une nouvelle traduction, d'un commentaire et d'une courte bibliographie.

2 L'introduction (p. 7 à 23) développe la définition de la prière au sens large comme « action verbale destinée à établir une relation avec le divin » et rappelle les tentatives de classifications, tant antiques que modernes, de la prière. L'accent est mis sur le caractère codifié de cette prière païenne, qui ne laisse presque pas de place à l'improvisation, ainsi que sur la gestuelle (p. 13-16), elle aussi très formalisée. Le relevé des schémas d'argumentation da quia dedi, da ut dem, da quia dedisti se retrouvera dans une trentaine de commentaires. L'index des sujets permet de retrouver les prières correspondant à ces schémas, auxquels s'ajoutent da quia non dedisti (2 exemples) et do 
ut des (6 exemples). Les auteurs font le choix judicieux d'insister sur le contexte littéraire et historique qui a produit la prière, prêtant attention à la rhétorique de l'époque, au genre, au but recherché. Rappelant l'existence d'autres corpus de prières, ils trouvent peu satisfaisantes les oppositions que ces ouvrages proposaient entre prière publique et prière privée, ou entre caractère codifié et poésie ou rhétorique.

Ils choisissent donc délibérément de juxtaposer tous les genres, depuis les inscriptions jusqu'aux vers des épopées ou des comédies, et adoptent pour ce faire un plan chronologique, conservant cependant la distinction entre textes grecs (101 textes, numérotés de G1 à G101, traduits et commentés par B. Laurot) et textes latins (numérotés de L1 à L89, traduits et commentés par F. Chapot). Ce qui n'est pas sans poser problème, reconnaissent-ils, quand un texte supposé archaïque (comme des formules d'évocation ou de dévouement) est rapporté par un auteur tardif, comme Macrobe. De même, pour le chant des arvales, donné dans sa transcription de $218 \mathrm{apr}$. J.-C., et qui est censé être plus ancien. C'est ainsi que le texte L1 propose des fragments des chants des saliens, cités par Varron et Terentius Scaurus; L2, le chant des arvales d'après le protocole de 218 ; L3, une prise d'auspices d'après Varron; L4, une litanie ancienne d'après Aulu Gelle; L5, une formule d'évocation d'après Macrobe; L6, une formule de dévouement d'après Macrobe et L7 à L13, des extraits de Plaute, plus anciens textes littéraires cités. Les six premiers extraits ont un incontestable parfum archaïque, mais comme ils sont des reconstitutions ou reconstructions qui datent, au plus tôt, de la fin de la République, ne pouvait-on pas les laisser figurer à la date de leur auteur? Il s'agit là d'une spécificité remarquable des textes choisis côté latin, puisque côté grec le classement chronologique n'a pas suscité de telles hésitations.

La séparation entre textes grecs et textes latins reste très commode, mais on regrettera l'absence de Plutarque (Vies parallèles, Questions romaines) ou de Denys d'Halicarnasse et de prières (archaïques ?) latines traduites en mots et réalités grecques par ces érudits.

Les auteurs étaient cependant conscients des limites de cette nouvelle proposition de classement et s'efforcent de les corriger par de nombreuses invitations aux rapprochements de textes (étudiés ou non dans le corpus) par-delà les siècles et d'une section à l'autre par-delà la langue. Une table chronologique (p. 400-401) permet de comparer les dates et met les uns en face des autres, en deux colonnes, les textes analysés. Quant à la juxtaposition d'inscriptions, de textes littéraires de genres nobles ou parodiques, elle est pleine de sens et conduit à des comparaisons fructueuses, à la fois pour l'étude de la religion et celle de la littérature.

6 L'ouvrage est très commode, et propose pour chaque texte une courte introduction, le texte dans sa langue originale, une traduction nouvelle (à de rares exceptions près), un commentaire plus ou moins développé selon la difficulté du texte, et tiré d'une courte bibliographie (voire discographie en G85, pour le péan processionnel à Apollon de Delphes) qui s'arrête à 1999 (et quelques ouvrages à paraître au-delà, comme les Actes du XXXIIIe Congrès international de l'APLAES, Clermont-Ferrand, 26-28 mai 2000, cités en L1 et L33, et désormais parus aux Presses de l'Université Blaise Pascal, ClermontFerrand, en 2001). La date tardive de ce compte rendu permet d'ajouter entre autres, dans une bibliographie qu'il faudrait pouvoir garder à jour, l'article d'A. Barchiesi sur le carmen saeculare d'Horace: "The uniqueness of the carmen saeculare and its tradition " (in T. Woodman et D. Feeney, Traditions and contexts in the poetry of Horace, Cambridge University Press, 2002). 
7 Le texte proposé en latin ou en grec tient compte, pour les textes les plus difficiles, de corrections postérieures à l'édition choisie (exemples côté latin: L1, L5, L15), ou, du moins, signale ces reconstructions (ex. L1, L3). Pour ce qui est de la traduction, presque toujours personnelle, les auteurs ont choisi, en suivant un projet de recherche sur les épiclèses, de traduire ces dernières. C'est ainsi qu'Apollon Sminthée devient, en G1, Apollon Destructeur de Mulots - épiclèse typiquement mysienne, dit le commentaire, renvoyant à la bibliographie. En L15, Jupiter Dapalis devient Jupiter hôte du sacrifice, traduction peut-être moins heureuse que celle de daps, rendu par " repas sacrificiel». La difficulté qu'il y a de tout traduire peut se lire dans le texte suivant, L16, où strues et fertum sont tous deux traduits par gâteau, avec, entre parenthèses, le mot latin (mais s'agit-il vraiment de gâteaux ?) ; en L18, ils ne sont plus traduits. Pour ce problème et un commentaire fourni des prières de Caton (L15-L18), on peut ajouter à la bibliographie le récent livre de John Scheid, Quand faire, c'est croire (Aubier, 2005), où tout un chapitre (p. 129-160) est consacré à l'analyse de ces textes.

8 Le commentaire, après des notes explicatives, insiste surtout sur la rhétorique de la prière en analysant la structure, le style, le choix des mots, leur mise en valeur dans les vers. Il s'accorde ainsi pleinement avec le titre de la collection, et peut être approfondi par la bibliographie.

9 L'annexe propose des textes latins (L90 à L98) et grecs (G102 à 108) sur la prière (typologie, textes philosophiques et moraux), traités selon le même principe que les précédents (traduction, rapprochements, orientations bibliographiques).

10 Un premier index permet de retrouver les sujets traités, tant le contenu ou le but de la prière (apostrophe, appel), que diverses remarques qui appartiennent au domaine rhétorique (ablatif absolu, apposition, assonance). C'est là qu'on trouvera aussi tous les noms des dieux invoqués. Sont ensuite présentés les auteurs et textes anciens, et enfin, les mots grecs et latins des prières qui ont fait l'objet d'un commentaire.

11 Un ouvrage, donc, qui est un bon outil, très pratique, et que l'on peut consulter de diverses manières, soit ponctuellement pour trouver un premier commentaire d'un texte précis, soit pour le lire dans son intégralité. La lecture peut alors se faire dans l'ordre chronologique des prières, ou en suivant les conseils donnés par les propositions de rapprochements, ou l'inspiration des mots-clés.

\section{AUTEURS}

\section{MARIE-KARINE LHOMMÉ}

Université Lumière (Lyon II). 